\title{
Robogami: a Fully Integrated Low-Profile Robotic Origami
}

\author{
Amir Firouzeh \\ Email: amir.firouzeh@epfl.ch \\ Jamie k. Paik \\ Email: jamie.paik@epfl.ch \\ Reconfigurable Robotics Laboratory \\ Department of Manufacturing and Robotics \\ EPFL \\ Lausanne, Switzerland
}

\begin{abstract}
To have an intelligent robotic system that can react to un-programmed tasks and unforeseen environmental changes, robots require augmented "softness". Robogami, a low-profile origami robot, addresses softness intrinsically (material-wise) and extrinsically (mechanism-wise) with its multi-Degree-of-Freedom rigid tile arrays with soft folding areas driven by embedded actuators. The unique hardware of the Robogami and its sub-millimeter thick construction enables diverse transformations as those achievable by the paper origami. The presented Robogami shows the first fully integrated version that has all the essential components including a micro-controller within a thin sheet. Construction of this robot is possible by using precise planar fabrication methods often used for micro system fabrications that has made prototyping of custom made robots rather low-cost and fast. In this research, we aimed at expanding the capabilities of Robogamis by embedding bi-directional actuation, sensing, and control circuit. To assess the performance of the proposed sensors and actuators, we report on the performance of these components in a single module and in the Crawler robot consisting of four modules as legs.
\end{abstract}

\section{Introduction}

Demanding new applications for robots often include interaction with unknown and ever altering environment that require softer and more compact systems [1] in order to meet the safety and reliability criteria in different applications such as search and rescue [2,3] or rehabilitation [4]. There are two main research streams toward achieving this softness: one is to fabricate the whole structure including the body and the actuators with materials that are inherently soft $[2,5]$ and the other is to make systems with many Degrees-of-Freedoms (DoFs) that consist of stiff parts with soft joints [6]. Having finite DoF, analysis and control of the latter is more feasible. Moreover, new methods for micro- fabrication makes miniaturization and hence the number of DoFs for such systems seemingly limitless. In these systems the resolution and the folding pattern for multiple folds of the robot are decided by the application.

Robotic Origamis (Robogamis) are low-profile, sheetlike robots that achieve softness and reconfigurability through multiple DoFs. In Robogami, the stiff tiles are connected in folding areas by low profile actuators that are inherently soft. Folding 2D patterns into 3D structures is an accurate and fast way of robot fabrication [7-10]. Our version of the Robogami, however, is a system that not only can make 2D to 3D transformation [11,12], but also includes actuators, sensors and circuitry [13]. Such a system can autonomously transform itself into its final shape and start performing the desired task. The origami structures that make up the underlying mechanisms in these robots can theoretically transform the shape of the body into any 3D shell using predetermined crease patterns [14]. In reality, this universality theory is limited by finite thickness and capabilities of the embedded actuators and sensors.

Softness in Robogami comes from its large number of DoFs: to achieve a high resolution, and hence DoF, the actuators should be small and have a low-profile for maintaining sub-millimeter thickness, they should also have sufficient energy density and range of motion to achieve the desired transformations. The Shape Memory Alloy (SMA) torsional actuator is a proper choice that satisfies this long list of requirements [15]. The large range of motion and blocked torque of these actuators, have already made them a popular choice for meso-scale robots [16-20]. In our design, two antagonistic SMA sheet actuators are used to make bidirectional folds in the Robogami.

Most studies on Robogami are only concerned with the folded and unfolded states that are achievable without feedback from the bending angle [12,21]. However, a Robogami, as any functional multi-DoF robotic system, requires feed- 


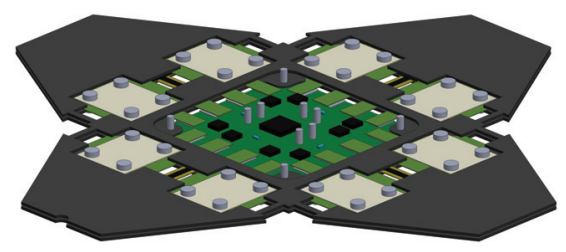

(a)

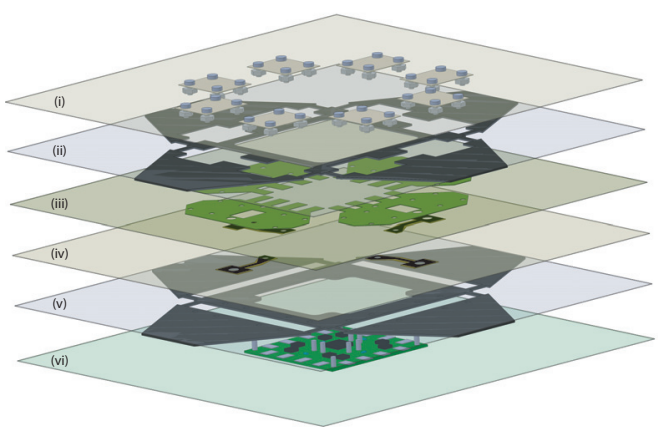

(b)

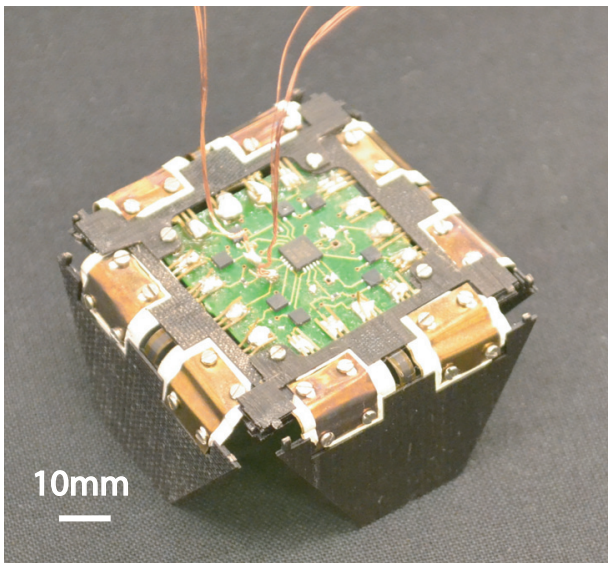

(c)

Fig. 1: The Crawler robot: (a) model, (b) exploded view of the functional layers (actuators are in layer (i). Layers (ii) and (v) are glass fiber layers that make the body. Layer (iii) contains the heaters and layer (iv) contains the sensors. Layer (vi) is the controller circuit.), and (c) fabricated robot

back from the bending angle on each fold. Previously, silicone-rubber micro-channels filled with conductive liquid [22,23] and conductive silicone [13] were considered as curvature sensors for Robogami. The main problem with the micro-channel sensors is their lack of robustness and difficulty of fabrication. For the conductive silicone sensors, the complex dynamic response [24] and the hysteresis in the sensor response which is dependent on the deformation rate is the main problem preventing an accurate feedback control [13]. So we have turned to another type of sensors based on piezo-resistive materials [25]. These sensors are easily customizable and their performance is not affected much by their size or shape. For curvature sensors in Robogami, we have chosen sensors made from printed carbon ink on polyimide layer [26]. These are more robust and have fewer problems with the dynamics of the filler material.

The main contribution of this paper is expanding the capabilities of the Robogamis by embedding bidirectional actuation, customizable curvature sensors, and control circuit. Here, we present the design and details of each of these elements and then study their overall performance in a Crawler robot made of 4 bending modules as legs. Figure 1 presents the design of this robot. The components embedded in it are presented in the exploded model in figure $1 \mathrm{~b}$.

In the next section, fabrication process of the Robogami is presented. Next, we will present the actuator and the sensor design parameters, their characterization, and their performance in a single module. In section IV, we present the result of successful interaction between different components in a Crawler robogami robot to achieve locomotion. Finally, section VI presents the conclusion of this work and the future steps.

\section{Fabrication of Robogami}

Robogamis are semi $2 \mathrm{D}$ robots that, depending on the application, might have different components. The main feature of these robots is their layer by layer fabrication method which enables us to process different functional layers separately and then stack them together. Many Origami based robots have been developed with different components to fulfill different tasks (basic as 2D to 3D transformation for robot body fabrication [7-10] or complicated as locomotion [27]). In the present design we aimed at making a Robogami structure independent as possible by embedding actuators, heaters, curvature sensors, and control circuit into glass fiber body layers (figure 1). These component are all processed individually and stacked together. Then in the integration step, the resin from Pre-impregnated glass fiber bonds all the layers together under pressure and heat. The cure temperature of the resin is $110^{\circ} \mathrm{C}$ which is acceptable for all components except for the actuators which have lower transition temperature. So the actuators are mounted on the cured structure using bolt and nuts in the last step.

As mentioned, in this design of Robogami, controller circuit is embedded in the robot. A micro controller (Attiny 1634) decides on the control sequence steps based on the feedback from the curvature sensors (the stepping sequence of the robot will be studied later). The command from the micro controller is amplified through a transistor and the output from the transistor is connected to the heaters that activate SMA actuators. Embedding the controller circuit makes the electrical connections of the robot much simpler and more robust and is a step toward making a completely autonomous Robogami structures.

For the body layers, the only process before integration is cutting the desired pattern which is done using a laser 
micro-machining tool [13]. As mentioned, sheet SMA actuators were chosen as actuators in this design of Robogami. These actuators needs to be annealed. So, after cutting, they are formed and get annealed in the desired shape (the annealed shape of the actuators will be discussed shortly). SMA actuators are heat activated and since self activation by passing current is not possible for the sheet actuators, we need an external heater layer. Here we used Polyimide backed Inconel (Ni-Cr alloy) sheet for producing the heaters. The fabrication of the heaters starts with covering the Inconel with etchant resist and then burning a serpentine pattern on the resist. This serpentine path makes large enough resistance to act as heater in the tile where it is in contact with the actuator. Then a mesh structure (similar to the mesh introduced for stretchable circuit in [22]) is cut through all layers in the folding area which makes a stretchable connection between the heaters on the two tile areas. Next, the outline of the heaters is cut and it is placed in the etchant tank for removing the Inconel along the serpentine cuts. Finally, the etchant resist is cleaned in acetone bath. To facilitate the connection of the sensors and the circuit, we also accounted for connection pads for the sensors on the heater layer which connects the sensors to the circuit.

With the limitations on the size, the minimum radius of curvature and shape, the use of commercially available sensors were ruled out and it was imperative to develop curvature sensors to meet all the needs of Robogami. Among sensor options for Robogami (e.g. liquid-metal filled microchannels and carbon based piezo resistive materials), we have chosen the carbon ink curvature sensors. These sensors

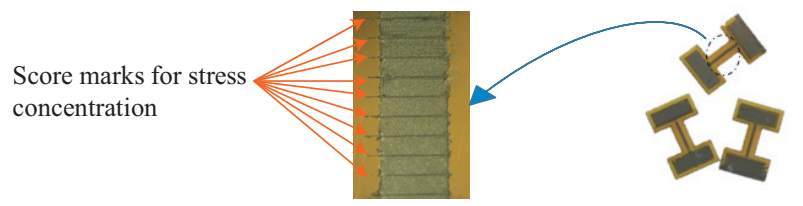

(a)
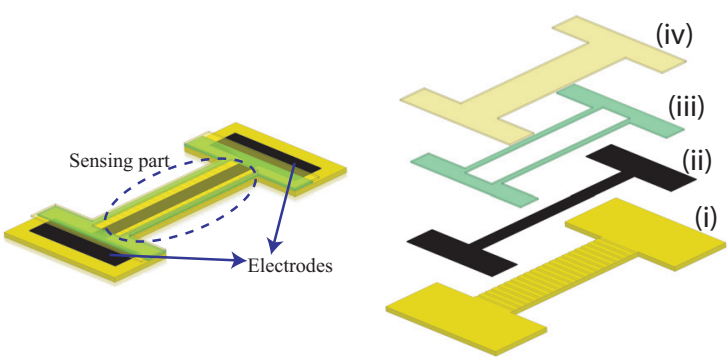

(b)

(c)

Fig. 2: (a) The curvature sensors and a close up of the sensing part to show parallel score marks. These marks increase the sensitivity in the direction parallel to them which is desired since we can increase the sensitivity to bending without affecting the sensitivity to twist. (b) The schematic of the layers of the sensor. (c) The exploded view presenting the base Polyimide layer (i), carbon ink layer (ii), hotmelt adhesive layer(iii), and the cover Polyimide layer. exhibit a high electrical resistance change under strain that is caused by micro cracks opening and closing in the carbon layer [26]. In order to increase the resistance change and to some extent regulate this effect, we have scored the polyimide substrate with the laser micro machining system introduced in [13]. Figure 2a shows these sensors and the score marks on the base Polyimide layer. Figure 2b, 2c presents the schematic of the sensor and an exploded view to show different layers in it. In these sensors, the score lines on the polyimide layer induces stress concentration which is used to amplify the resistance change in a desired direction. This is useful for reducing the reading error caused by the twist which will be discussed shortly. The cover Polyimide layer in $2 \mathrm{c}$ was added to protect the conductive path.

The fabrication process for these sensors starts with covering the surface of the base Polyimide layer (layer (i) in figure $2 \mathrm{c}$ ) with masking tape and cutting the outline of the deposition area and removing the tape in those areas. Next, the surface of the base Polyimide layer is scored with the laser beam down to one third of its thickness and a layer of carbon ink is deposited and the mask is removed (this leaves us with the carbon ink layer (ii) presented in figure $2 \mathrm{c}$ ). Then, the carbon layer is covered with a protective layer (Polyimide cover layer (iv) in figure 2c) which prevents scratches on the conductive layer. This layer is attached using a hotmelt adhesive sheet, which is cut to the right form with the laser (layer (iii) in figure 2c), to the base Polyimide layer in the heat press. Finally, the sensor outline is cut and it is attached to the electrodes designed on the heater layer (the heater layer also plays a role of intermediate conductive circuit between controller and the sensor). The overlay of the sensor and heater layer (the connection part) can be seen in figure $1 \mathrm{~b}$.

As mentioned above after preparing all the layers, they are integrated into a robotic sheet, that embeds all the functional layer, in a heat press. In the next 2 sections details of the characterization of the actuator and sensor layers will be presented.

\section{SMA torsional actuator for Robogami}

High torque to mass ratio of thin sheet SMA actuators makes them an attractive candidate for low profile and compact robots $[15,17]$. These actuators are also compatible with 2D layer-by-layer fabrication of Robogami and provide potential for easy integration in systems with multi-DoFs. For bidirectional actuation, we use two antagonistic actuators on each fold as presented in figure 3 .

Assuming a simple bending beam model, a uniform radius of curvature along the actuators, and neglecting the twist in the tiles, Eqn. (1) yields the torque in each SMA actuator.

$$
\left.\begin{array}{l}
\varepsilon=\left(\frac{y}{R}=\frac{y \theta}{L_{a}}\right) \\
\sigma=F(\varepsilon, T e m p)
\end{array}\right\} T=\int_{-t / 2}^{-t / 2} y(w \sigma d y)
$$

In Eqn. (1), $t$ and $w$ are thickness and width of the actuator. $\varepsilon$ and $\sigma$ are stain and stress, $y$ is the distance from the 


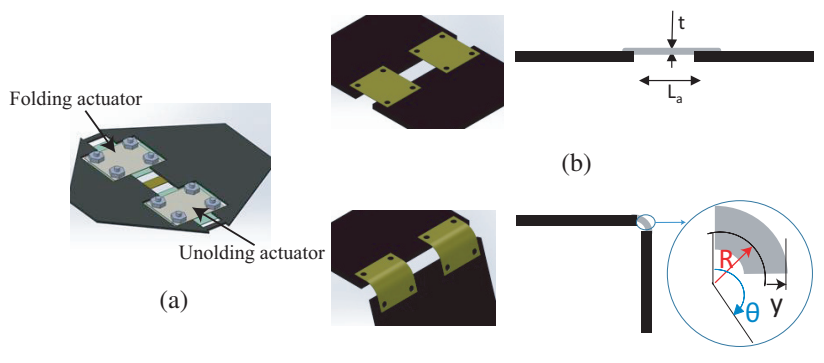

(c)

Fig. 3: (a) A module with antagonistic bending actuators. one of the actuators have its annealed shape in folded state while the other have unfolded annealed shape. By activating each of the two we can transform between the folded and unfolded states. (b) Unfolded module and the schematic of its side view. (c) folded state and its side view schematic. In the side views the tiles are shown in black and the gray parts represent the actuators.

neutral plane, $R$ is the radius of curvature, $L_{a}$ is the length of the actuator and $\theta$ is the bending angle (figure 3 presents the geometric parameters governing the torque and bending angle relation). To evaluate this equation, stress-strain data $F(\varepsilon, t e m p)$ is required. Here, we used data from [28]. Since $F(\varepsilon, t e m p)$ is dependent on many factors such as annealing process, number of actuation cycles and grain structure of the metal, which are different from case to case. So, this study, using the data from [28], will not give us accurate numbers for torque, but it can provide us with an understanding of the important design parameters and the overall behavior of SMA actuators in antagonistic arrangement. Figure 4

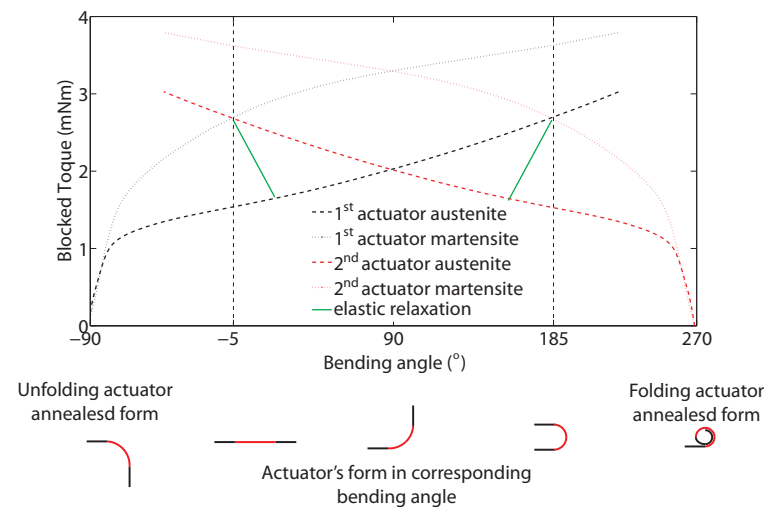

Fig. 4: Torque in the folding and unfolding actuators. Blocked torque of the unfolding actuator is presented by black lines which is zero at $-90^{\circ}$ that corresponds to the memory shape of the actuator (the schematics in the figures presents the corresponding shape of actuators in each bending angle). Based on the temperature of the actuators (the phase of the material), each bending angle corresponds to two values: one for active (heated) and one for passive (cold) actuator. For folding actuator (red lines), the memory shape (zero torque point) is at $270^{\circ}$ and its trend is similar as before. There are two equilibrium points that correspond to the intersection of the blocked torque of an activated (heated) actuator with the other actuator in inactive (cold) state. After reaching the equilibrium point and cutting off the current, the equilibrium point stars to shift along the green lines (elastic relaxation of the passively deformed actuator) till reaching the new equilibrium where both actuators are cold.

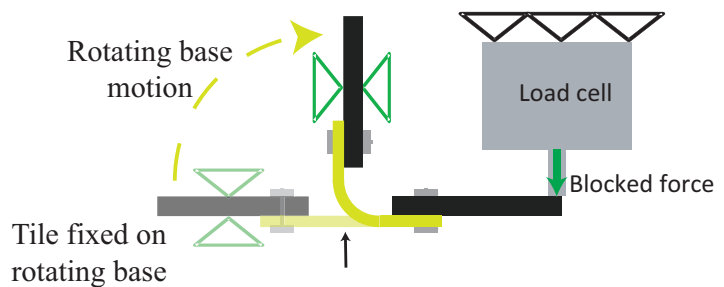

Fig. 5: Schematic of the test setup for measuring blocked torque of SMA actuators in their whole range of motion

presents the torque generated by the activated SMA actuator and the torque that is needed to deform the passive actuator as a function of the bending angle based on Eqn. (1).

In figure 4 , there are three lines for the blocked torque of each actuator. They correspond to the torque that the activated actuator (in Austenite phase) generates, the torque that is needed to deform the inactive actuator (in Martensite phase) and the elastic relaxation in the passive actuator around the equilibrium point when it is unloaded. The intersections of the lines corresponding to the Austenite phase of one actuator and the Martensite phase of the other determines the equilibrium angle of the fold with bidirectional actuation. When the current to the active actuator is cut off, it starts to cool down and the torque in it drops to the Martensite phase line. The torque in the passive actuator also drops along the unloading line till a new equilibrium point is achieved. The range of motion for a fold with bidirectional actuation is determined by the length of the actuator. According to the model, choosing the length to be $4.5 \mathrm{~mm}$ (for $0.1 \mathrm{~mm}$ thick actuators), the range of motion will slightly be higher than $180^{\circ}$ (figure 4 ). Here we have chosen the annealed position of the actuators in a way to get one equilibrium point on unfolded state $\left(0^{\circ}\right)$ and another one on the folded state $\left(180^{\circ}\right)$. We also assumed $8 \%$ as maximum allowable strain for the actuator in the equilibrium points.

The design of the actuators (their dimensions and annealed shapes) are based on the result of the theoretical study presented above. In the remaining of this section, we study the performance of the actuators through experiments and more accurately investigate the accessible range of motion in a fold with bidirectional actuation and also the response time and the blocked torque of the actuators.

When activated without any opposing force, SMA actuators have high fidelity to their memory shape in repeated cycles. But under load they suffer from memory loss. Many factors such as the maximum strain, the opposing force and the temperature gradient play a part in this memory loss. Also, the range of motion of the actuators in antagonistic arrangement is smaller than the bending angle of the annealed shape of each actuator. This is caused by the opposing force needed for deforming the other actuator. Because of this and also the memory loss, SMA actuators should be annealed in deformations larger than the goal angles. We have chosen the annealing shape of the folding actuators as a cylinder with diameter close to $1.5 \mathrm{~mm}$ (the folding actuator annealed shape is presented in figure $6 \mathrm{a}$. For the unfolding actuator, the an- 


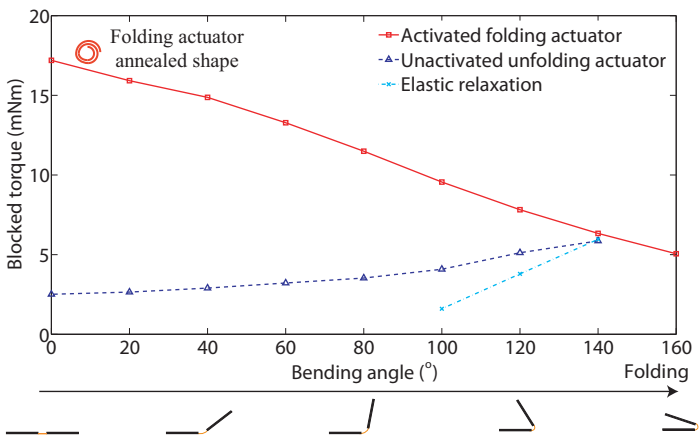

(a)

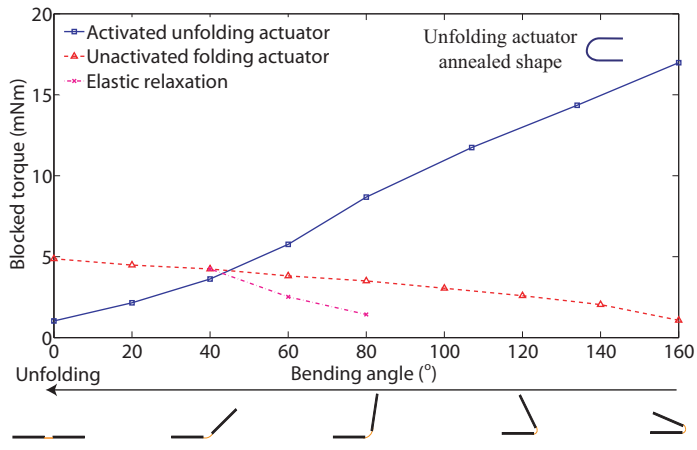

(b)

Fig. 6: Result of the blocked torque tests which corresponds to the theoretical result presented in figure 4. (a) Torque generated by the actuators in motion toward $180^{\circ}$ (the folded state) with the folding actuator activated and passively deformed unfolding actuator. (b) Torque generated by the actuators in motion toward $0^{\circ}$ (the unfolded state) with the unfolding actuator activated and passive folding actuator. The elastic relaxation in both plots corresponds to unloading of the passive actuator which happens when the current to the active actuator is cut off and it starts to cool down and its blocked torque decreases.

nealed shape was a half cylinder with the radius of curvature close to $3 \mathrm{~mm}$ (the actuator annealed shape is presented in figure 6b). These annealed shapes produce large enough bending angles to enable the crawler to walk.

In the Crawler, the resisting torque against deformation comes from the antagonistic actuator and also from the weight of the structure. So other than the accessible range of motion, we need to obtain a measure of the blocked torque of the actuators. Figure 5 presents the setup used for blocked force tests. The rotating base in this setup lets us measure the blocked forces in different bending angles.

Because of the aforementioned memory loss, actuator's blocked torque depends on its activation history. When the actuator is activated while kept under maximum deformation, the torque generated later in lower strain would be smaller. To have a conservative estimation of the blocked torque of the actuators, we activated them in the maximum deformation and step by step relaxed the strain (it gives a conservative result because in the antagonistic arrangement under large stain, the opposing force of the other actuator, and hence memory loss, is much smaller). Figure 6 presents the results of the characterization test for folding and unfolding actuators. The blue lines present the torque in the unfolding actuator (with the annealed shape of $\left(-180^{\circ}\right)$ and the red lines present the torque in the folding actuator (the annealed shape of complete cylinder). This figure also presents the elastic relaxation of the inactivated actuator around its equilibrium point. The small slope of this elastic relaxation implies that when the driving actuator starts to cool down there will be a rather large relaxation and change in the position. This makes the feedback control for Robogami modules a necessity.

The conservative characterization of the blocked torque predicts around $100^{\circ}$ range of motion for the fold with bidirectional actuation (figure 6). This is enough for many applications. If larger range of motion is required, one could use a thinner or longer actuators (according to Eqn. (1)). Both of these decrease the strain level for a given bending angle).

In the blocked torque tests, with heating power of 1.8
$\mathrm{W}$ it takes around $15 \mathrm{~s}$ for actuators to generate maximum torque which is not in agreement with initial expectations based on [13]. To investigate the cause for this slow response we simulated the transient thermal phenomenon. Neglecting radiation, the heat transfer equation for SMA is as follow:

$$
\rho C \dot{T}+\Delta u \dot{Z}=q_{\text {contact }}+q_{\text {convection }}+\gamma(T) \Delta T
$$

In this equation, $\rho, C, \Delta u, \gamma$ are density, thermal capacity, latent heat of phase transition, and thermal conductivity coefficient respectively. $T$ and $Z$ are temperature and the

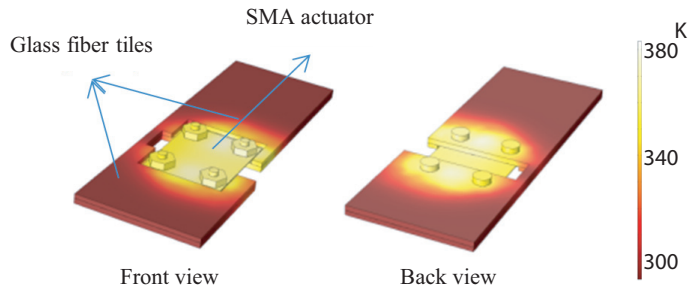

(a)

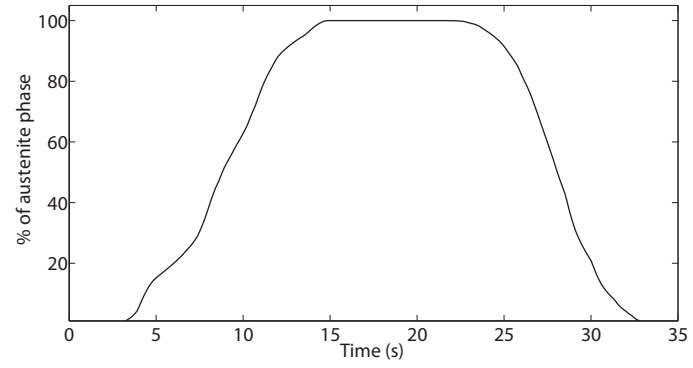

(b)

Fig. 7: Thermal analysis for an SMA torsional actuator. (a) The temperature gradient in the actuator and the tiles, corresponding to the instance that all SMA material has transformed to Austenite phase. (b) SMA phase transition versus time. 
mass in Austenite phase. Heat transfer between SMA and its surrounding happens through natural convection $\left(q_{\text {convection }}\right)$ and contact with other solid surfaces $\left(q_{\text {contact }}\right)$. Here we assumed zero thermal resistance for contact between different layers. As presented in this equation, the input heat to each element (right hand side of the Eqn. (2)) divides to two portions. One increasing the temperature $(T)$ and the second causes the phase transition $(Z)$.

For other components (glass fiber layers, heater layer, bolts and nuts) the governing equation is the same as Eqn. (2) with the difference that there is no latent phase transition heat and the temperature dependency of thermal conductivity of the material is negligible (unlike SMA where we have a large change after phase transition).

For SMA thermal characteristics, we have used the material properties from [29]. Figure 7 shows the result of this thermal analysis. As presented in figure 7a, because of the location of the heaters, lots of heating power is wasted on heating up the glass fiber layer. Initially, based on the glass fiber lower thermal conductivity (20 times less than that of the SMA) we have expected it to be insulating and to have little influence on the transient thermal response of the system. But as can be seen in figure 7a temperature rise in the glass fiber layer is substantial. The phase transition in the SMA actuator versus time (figure 7b) shows that nearly 15 $\mathrm{s}$ is needed for complete transition of the SMA to Austenite phase. In this plot, the slope change during heating is caused by latent heat of phase transition and also change in thermal conductivity of SMA in different phases.

To improve the actuator response time, there are two options: using more insulating layers or placing the heaters on the active part of the SMA actuators. By relocating the heaters, we also expect to decrease the rate of the memory loss in the actuators, due to more uniform temperature distribution.

\section{Flexible curvature sensor for Robogami}

To characterize the curvature sensor presented in Figure2, its resistance was measured for different bending angles. We used a voltage divider circuit to detect the resistance change in the sensors in the repeated cycles of fold-

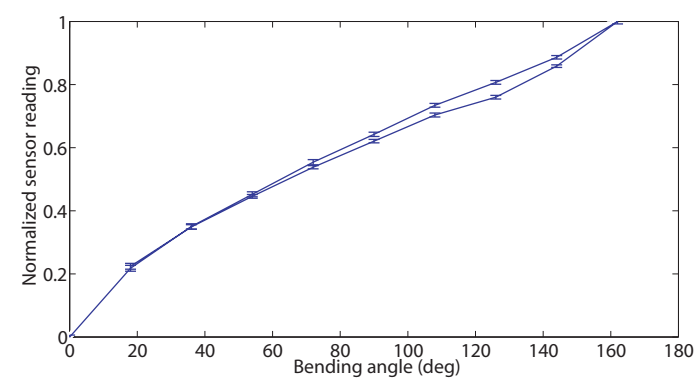

Fig. 8: Mean value and standard deviation for normalized readings of the sensor versus the bending angle in 50 cycles. The result shows very good repeatability and small hysterysis loop compared to conductive silicone based sensors previously suggested in [13].

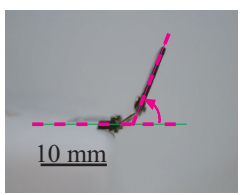

(a)

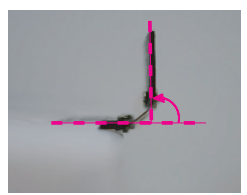

(b)

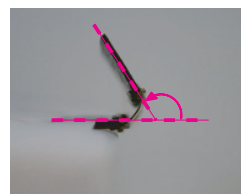

(c)
Fig. 9: Three control angles achieved using curvature sensor feedback with goal set to (a) $60^{\circ}$, (b) $90^{\circ}$, and (c) $120^{\circ}$

ing and unfolding which was performed with $18^{\circ}$ increments. Sensor readings in 50 cycles and the mean value as a function of bending angle is presented in figure 8 . We have normalized the readings with the maximum amplitude of voltage change in this figure. Other than robustness and improved repeatability, this plot presents another advantage of this sensors over conductive silicone-based sensors which is the smaller hysteresis loop. The average standard deviations for this sensor is $1.1^{\circ}$. This is an improvement compared to the previous results [13]. To sum up, sensors made of conductive ink track on polyimide sheet are more accurate, robust, and reliable. The only limitation on application of these sensors is that unlike conductive silicone based sensors, these are not stretchable which for the case of a curvature sensors is not important. The characterization result presented here is very brief and only covers quasi static readings in repeating cycles (which is also the case for the Crawler application). A more detailed study on transient response and drift in sensor readings as well as a model for explaining the response of the sensors is the topic for future studies.

In the characterization tests, we deformed the sensors in pure bending. In the case of bidirectional actuation, a small twist also occurs in the folding area. This is because of the torque in the passive actuator, which is a function of its temperature. This makes the shape of the sensor slightly different in the consecutive cycles. To study the effect of this twist on the sensor performance, here the accuracy of the sensor is measured in the feedback control of a module with bidirectional actuation. The test scenario is the same as what is needed for locomotion of the Crawler. Based on the desired bending angle and the current bending angle, an on-off controller with a small dead-band decides to activate the folding or unfolding actuators. Figure 9 presents snapshots of the three control angles $\left(60^{\circ}, 90^{\circ}\right.$, and $\left.120^{\circ}\right)$. We have studied feedback control in five cycles. The average of the standard variation for these three angles was $1.4^{\circ}$ which shows a very good accuracy. This is because of more repeatable readings from the sensors and the minimized effect of the twist error in the sensors with the polyimide backing.

\section{Robogami Crawler : four-legged mobile Robogami}

Robogami is a multi DoF, low profile robot that performs different tasks by reconfiguring its shape. Here, we use a mobile robot consisting of four folding modules (figure 1a) as a case study to assess the performance of the actuators, the sensors, and the overall design of the Robogami. 


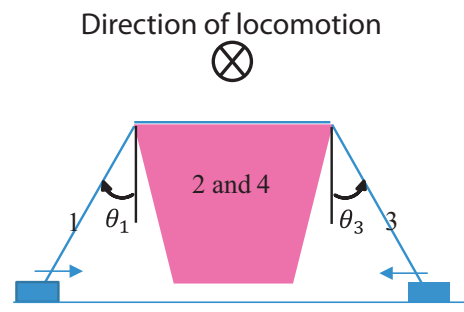

(a)

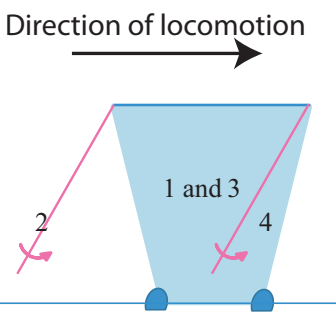

(b)

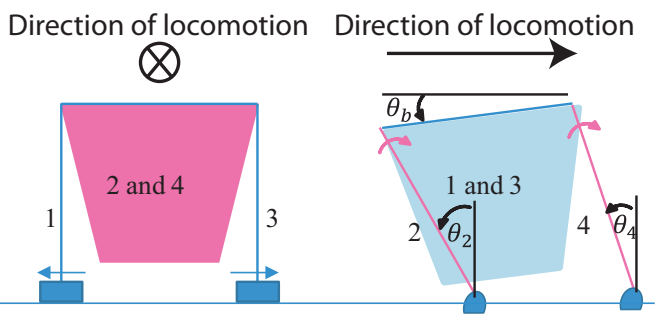

(c)

(d)

Fig. 10: The equivalent mechanisms for different phases of locomotion. (a-d) represent the phases number 1-4 introduced in Table 1 respectively. Figure (a) and (c) show the view of the robot from the back along the locomotion direction which presents the motion of active legs in these two phases (leg 1-3). Legs 2 and 4 that are presented in pink are inactive in these two states. Figure (b) and (d) show the view of the robot from the side and normal to the locomotion direction to present the motion of active legs in these two phases (leg 2-4). Legs 1 and 3 that are presented in blue are inactive in these two states.

In this section, we study torques needed for locomotion of the crawler robot and present the locomotion of the robot in experiment.

\subsection{Robogami Crawler: stepping sequence}

Here we present the stepping sequence of the Robogami crawler, evaluate the torque needed for its locomotion, and compare that to the blocked torque from characterization tests. There are four phases in the Crawler locomotion as presented in Table 1. The angles in this table are those between the legs and the center tile. Shaded cells in the table indicate legs that bear the weight of the robot in each phase while the other two legs have no contact with the surface and can move without any contact force. Figure 10 shows the corresponding mechanism for each phase.

Since the speed and acceleration of the robot are small (given the slow response of SMA actuators), here, we calculate the actuation torque necessary for locomotion in the quasi static state. The torque required in phase 2 and 3 are clearly smaller than the torque needed in phase 1 and 4 . So here we only study forces in phase 1 and 4 . We start with investigating the required torque in phase 4 which is presented in figure 10d. This figure shows a general case that corresponds to out of sync motion of leg 2 and 4 but to evaluate the torque needed for locomotion in this phase, we considered in sync motion of the two legs which is the ideal case for the locomotion $\left(\theta_{2}=\theta_{4}, \theta_{b}=0\right)$. The more general representation of the figure however, helps with explanation of the

Table 1: The goal angles for each leg in four phases of locomotion. The shadowed cells indicate the legs on the ground

\begin{tabular}{ccccc}
\hline & Phase 1 & Phase 2 & Phase 3 & Phase 4 \\
\hline Leg \# 1 & $90^{\circ}$ & $90^{\circ}$ & $45^{\circ}$ & $45^{\circ}$ \\
Leg \# 2 & $135^{\circ}$ & $45^{\circ}$ & $45^{\circ}$ & $135^{\circ}$ \\
Leg \# 3 & $90^{\circ}$ & $90^{\circ}$ & $45^{\circ}$ & $45^{\circ}$ \\
Leg \# 4 & $45^{\circ}$ & $135^{\circ}$ & $135^{\circ}$ & $45^{\circ}$ \\
\hline
\end{tabular}

slippage in phase 4 of locomotion which will be discussed shortly. For the normal forces in phase 4 we have:

$$
\begin{array}{r}
N_{2} L_{b}=\left(m_{b}+2 m_{l}\right) g\left(\frac{L_{b}}{2}+L_{l} \sin \theta_{2,4}\right) \\
+m_{1} g L_{l} \sin \theta_{2,4}+m_{l} g L_{b} \\
N_{4} L_{b}=\left(m_{b}+2 m_{l}\right) g\left(\frac{L_{b}}{2}-L_{l} \sin \theta_{2,4}\right) \\
-m_{l} g L_{l} \sin \theta_{2,4}+m_{l} g L_{b}
\end{array}
$$

Considering in sync motion and small acceleration, the horizontal force at at the contact point is assumed to be zero and the drive torque for this phase is calculated as:

$$
\tau_{2,4}=N_{2,4} L_{l} \sin \theta_{2,4}-m_{l} g \frac{L_{l}}{2} \sin \theta_{2,4}
$$

In Eqn. (3)-(5) $N$ and $\tau$ are the normal force, and the drive torque in each leg. $L_{1}, L_{b}$ are the leg and the center tile lengths which are $22 \mathrm{~mm}$ and $44 \mathrm{~mm}$, respectively. The dimensions of the robot were designed to prevent it from tripping. $m_{l}, m_{b}$ are the leg and center tile mass which are 0.9 $\mathrm{g}$ and $3.7 \mathrm{~g}$, respectively. Considering $90^{\circ}$ range of motion, the maximum torque in this phase of locomotion (Eqn. (5)) is $0.83 \mathrm{mNm}$.

In phase 1 of locomotion (figure 10a), the required torques for leg 1 and 3 are given as Eqn. (6).

$$
\begin{array}{r}
\tau_{1,3}=\frac{m_{b}+4 m_{l}}{2}\left(g L_{l} \sin \theta_{1,3}+\mu g L_{l} \cos \theta_{1,3}\right) \\
-m_{1} g L_{l} \sin \theta_{1,3}
\end{array}
$$

The required torque in phase 1 is dependent on the friction coefficient, $\mu$, which can be managed by choosing the right material at the point of contact. Because of the small 


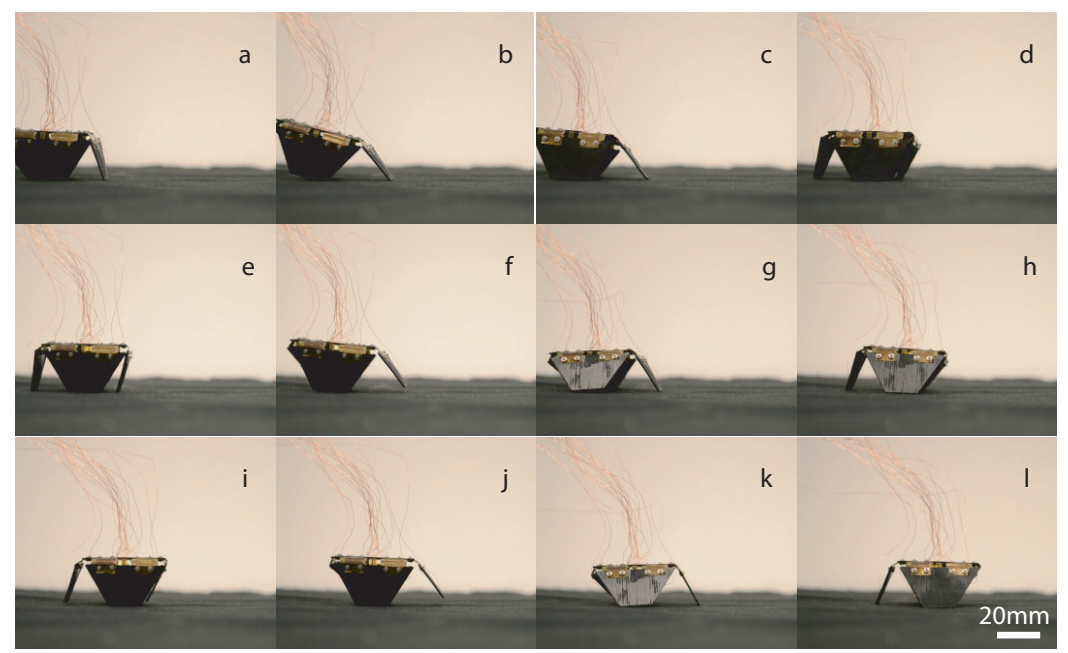

Fig. 11: The sequence of the motion of the Crawler. (e,i) show phase 1, (b,f,j) phase 2, (c,g,k) phase 3, and (d,h,l) phase 4 .

acceleration, even small friction forces can secure locomotion without any slip in phase 4 . So based on the design, the torque required for this phase can be made smaller than what we get for phase 4 (this torque is enough for motion on surface with friction coefficient of 0.65 in phase 1) which is an order of magnitude smaller than the torque in the passive actuator measured in blocked torque test (figure 6). Therefore, the deciding factor on range of motion of the joints in the Robogami crawler is the torque in the antagonistic actuators.

During phase 4 of the locomotion, the ideal step size for the robot is $2 L_{1} \sin \theta_{\max }$. But in the experiments, each step is smaller because of the slip between the legs and the surface. To investigate the reason for this, we have studied forces during this phase. If both legs move together, the friction force will push the robot forward. But what happens in the experiments is that one of the legs starts moving a little faster than the other. This causes the leg that is moving to push the body forward while the other one is resisting the locomotion. This out of sync motion results in a configuration similar to figure 10d. In general the leg closer to the center of mass has larger normal force so if the forward leg starts moving first, in the beginning and final part of the motion the normal force of the active leg (front in the beginning part and hind leg in the final part) will be smaller than the passive one and the active leg slides which means that the step size will be smaller than the ideal one.

On the other hand, if the hind leg starts moving first, active leg will always have the higher normal and friction force and the step size will be the same as the ideal one. At the moment we activate both legs at once and do not control the leg position before reaching the goal angle. This leads to out of sync motion and slip in some parts of motion and hence smaller step size.

\subsection{Robogami Crawler: locomotion}

In this section, the results of the experiment on the robot are presented. The controller decides the goal angles for each leg based on the phase of locomotion of the robot and using the feedback from the sensors, determines when the goal configuration is achieved and switches to the next phase. In each phase, based on the actual and desired goal angles, an on-off controller activates the actuators to reach the desired angle. Due to the fluctuation around the equilibrium point in each leg, reaching the goal angle on all legs might not happen at once. So, Each phase is considered complete if all legs have reached their goal angle once from the beginning of that phase. Figure 11 presents series of the snapshots taken from the Crawler as it moves three steps.

The series of the snapshots of figure 11 indicates that the bidirectional folds that are activated by the SMA actuators, feedback that is provided by the carbon ink printed sensors, the controller and the overall design of the Robogami are all effective. Also, the controller circuit successfully closes the loop using the sensor reading and generate the appropriate command. The locomotion of the robot is rather slow which can be alleviated in future, by moving the heaters to the active part of the actuators as suggested in [17]. In phase 4 of the locomotion, due to the relaxation in legs number 1 and 3 , these two legs touch the ground. This effect in addition to the out of sync motion makes the step size smaller and locomotion slower. Solving these problems will be a topic for the future studies.

\section{Conclusions and future work}

In this paper, the bidirectional actuation using SMA torsional sheet actuators, feedback control using carbon ink based sensors and overall design for the Robogami were presented and the performance of the proposed components were studied in a single module and a mobile robot consisting of four modules as legs.

The flexible curvature sensors were fabricated by printing carbon ink tracks on polyimide sheet and embedded in the Robogami modules. To improve the response of the sensor, the surface of the polyimide layer was scored with laser 
(prior to applying the carbon ink) in order to make lines of stress concentration in the carbon layer. These sensors are very robust and they have small hysteresis in their response. The average standard deviations for this type of sensor in 50 cycles of folding and unfolding was measured to be $1.1^{\circ}$. Also the average of the standard deviation for reaching three goal angles $\left(60^{\circ}, 90^{\circ}, 120^{\circ}\right)$ in feedback control of one module was $1.4^{\circ}$. These results show an improvement over what was achieved previously using other types of sensors for Robogami.

In this research, the Robogami Crawler consisting of four folding modules was designed and prototyped to assess the performance of actuators, sensors, and layer by layer fabrication of the Robogami. The blocked torque of the actuators was measured (5-16 $\mathrm{mNm}$ in the whole range of motion) to ensure that they are capable of producing enough torque $(0.83 \mathrm{mNm})$ for carrying the Crawler's weight. The experimental results confirmed that the actuators can provide large enough range of motion in each leg, the sensors can provide robust and reliable feedback for angle control in legs and finally the design for the circuit and other parts can endure high deformations. In more complicated Robogamis with a large number of folding areas, attaching the wires for transferring the data and the order between the controller and the robot would not be feasible. Therefore, a control circuit was designed and embedded in the Robogami which gets the feedback from the sensors and produces the command for the actuators accordingly. The Robogami Crawler presented here is the first example toward a fully integrated planar robotic system that can transform its shape using components embedded in it. In future, by embedding the power source such a system can become completely autonomous. The work presented here paves the way toward fabrication of more complex Robogamis, with more folding areas (DoF), capable of performing more complicated tasks in future.

\section{Acknowledgements}

This research is supported by Swiss National Centers for Competence in Research (NCCR) in Robotics.

\section{References}

[1] Kim, S., Laschi, C., and Trimmer, B., 2013. "Soft robotics: a bioinspired evolution in robotics". Trends in Biotechnology, 31(5), pp. 287-294.

[2] Firouzeh, A., Ozmaeian, M., Alasty, A., and zad, A. I., 2012. "An ipmc-made deformable-ring-like robot". Smart Materials and Structures, 21(6), p. 065011.

[3] Matsuda, T., and Murata, S. "Stiffness distribution control - locomotion of closed link robot with mechanical softness". In Robotics and Automation, 2006. ICRA 2006. Proceedings 2006 IEEE International Conference on, pp. 1491-1498.

[4] Song, Y. S., Sun, Y., van den Brand, R., von Zitzewitz, J., Micera, S., Courtine, G., and Paik, J., 2013. "Soft robot for gait rehabilitation of spinalized ro- dents". In Intelligent Robots and Systems (IROS), 2013 IEEE/RSJ International Conference on, pp. 971-976.

[5] Huai-Ti, L., Gary, G. L., and Barry, T., 2011. "Goqbot: a caterpillar-inspired soft-bodied rolling robot". Bioinspiration and Biomimetics, 6(2), p. 026007.

[6] Hines, L., Arabagi, V., and Sitti, M., 2012. "Shape memory polymer-based flexure stiffness control in a miniature flapping-wing robot". Robotics, IEEE Transactions on, 28(4), pp. 987-990.

[7] Felton, S., Tolley, M., Onal, C., Rus, D., and Wood, R., 2013. "Robot self-assembly by folding: A printed inchworm robot". In Robotics and Automation (ICRA), 2013 IEEE International Conference on, pp. 277-282.

[8] Noh, M., Kim, S.-W., An, S., Koh, J.-S., and Cho, K.J., 2012. "Flea-inspired catapult mechanism for miniature jumping robots". Robotics, IEEE Transactions on, 28(5), pp. 1007-1018.

[9] Martinez, R. V., Fish, C. R., Chen, X., and Whitesides, G. M., 2012. "Elastomeric origami programmable paper-elastomer composites as pneumatic actuators". Advanced Functional Material, 22, pp. 1376-1384.

[10] Onal, C. D., Wood, R. J., and Rus, D. "Towards printable robotics: Origami-inspired planar fabrication of three-dimensional mechanisms". In on Robotics and Automation (ICRA), 2011 IEEE International Conference, pp. 4608-4613.

[11] An, B., and Rus, D. "Programming and controlling self-folding robots". In Robotics and Automation (ICRA), 2012 IEEE International Conference on, pp. 3299-3306.

[12] Paik, J., An, B., Rus, D., and Wood, R. J., 2011. "Robotic origamis: self-morphing modular robots". Proceedings of the 2nd International Conference on Morphological Computation.

[13] Firouzeh, A., Sun, Y., Lee, H., and Paik, J., 2013. "Sensor and actuator integrated low-profile robotic origami". In Intelligent Robots and Systems (IROS), 2013 IEEE/RSJ International Conference on, pp. 49374944.

[14] Benbernou, N., Demaine, E. D., Demaine, M. L., and Ovadya, A., 2009. "A universal crease pattern for folding orthogonal shapes". arXiv preprint arXiv:0909.5388.

[15] Paik, J., Hawkes, E., and Wood, R., 2010. "A novel low-profile shape memory alloy torsional actuator". Smart Materials and Structures, 19(12), p. 125014.

[16] Torres-Jara, E., Gilpin, K., Karges, J., Wood, R. J., and Rus, D., 2010. "Compliant modular shape memory alloy actuators". Robotics and Automation Magazine, IEEE, 17(4), pp. 78-87.

[17] Paik, J. K., and Wood, R. J., 2012. "A bidirectional shape memory alloy folding actuator". Smart Materials and Structures, 21(6), p. 065013.

[18] Nishida, M., Tanaka, K., and Wang, H. O. "Development and control of a micro biped walking robot using shape memory alloys". In Robotics and Automation, 2006. ICRA 2006. Proceedings 2006 IEEE International Conference on, pp. 1604-1609. 
[19] Matsumoto, Y., Nakanishi, H., and Hirai, S., 2008. "Rolling locomotion of a deformable soft robot with built-in power source". In Proc. 11th Int. Conf. Climbing and Walking Robots and the Support Technologies for Mobile Machines (CLAWAR 2008), pp. 365-372.

[20] Mingyen, H., McMillan, A. B., Simard, J. M., Gullapalli, R., and Desai, J. P., 2012. "Toward a meso-scale sma-actuated mri-compatible neurosurgical robot”. Robotics, IEEE Transactions on, 28(1), pp. 213-222.

[21] Hawkes, E., An, B., Benbernou, N. M., Tanaka, H., Kim, S., Demaine, E. D., Rus, D., and Wood, R. J., 2010. "Programmable matter by folding". Proceedings of the National Academy of Sciences.

[22] Paik, J. K., Kramer, R. K., and Wood, R. J. "Stretchable circuits and sensors for robotic origami". In Intelligent Robots and Systems (IROS), 2011 IEEE/RSJ International Conference on, pp. 414-420.

[23] Kramer, R. K., Majidi, C., Sahai, R., and Wood, R. J. "Soft curvature sensors for joint angle proprioception". In Intelligent Robots and Systems (IROS), 2011 IEEE/RSJ International Conference on, pp. 19191926.

[24] Lacasse, M. A., Duchaine, V., and Gosselin, C. "Characterization of the electrical resistance of carbon-blackfilled silicone: Application to a flexible and stretchable robot skin". In Robotics and Automation (ICRA), 2010 IEEE International Conference on, pp. 4842-4848.

[25] Rosset, S., and Shea, H., 2013. "Flexible and stretchable electrodes for dielectric elastomer actuators". Applied Physics A, 110(2), pp. 281-307.

[26] Avalon, T. D., Fuller, R., Gentile, C. T., Goodman, S., Hall, T., and Wallace, M., 1992. Angular displacement sensors, Feb. 11. US Patent 5,086,785.

[27] Wood, R. J., Avadhanula, S., Sahai, R., Steltz, E., and Fearing, R. S., 2008. "Microrobot design using fiber reinforced composites". Journal of Mechanical Design, 130(5), pp. 052304-052304. 10.1115/1.2885509.

[28] Lu, Z. K., and Weng, G. J., 1998. "A self-consistent model for the stressstrain behavior of shape-memory alloy polycrystals". Acta Materialia, 46(15), pp. 54235433.

[29] Nitinol technical properties. http://jmmedical.com/resources/221/NitinolTechnical-Properties.html. 